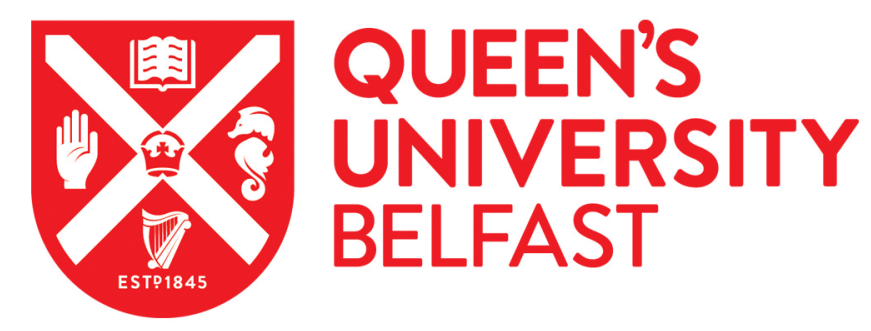

\title{
Evaluation of crystallographic strain, rotation and defects in functional oxides by the moiré effect in scanning transmission electron microscopy
}

Naden, A., O'Shea, KJ., \& MacLaren, DA. (2018). Evaluation of crystallographic strain, rotation and defects in functional oxides by the moiré effect in scanning transmission electron microscopy. Nanotechnology, 29(16), [165704]. https://doi.org/10.1088/1361-6528/aaae50

Published in:

Nanotechnology

Document Version:

Peer reviewed version

Queen's University Belfast - Research Portal:

Link to publication record in Queen's University Belfast Research Portal

Publisher rights

Copyright 2018 IOP. This work is made available online in accordance with the publisher's policies. Please refer to any applicable terms of use of the publisher.

\section{General rights}

Copyright for the publications made accessible via the Queen's University Belfast Research Portal is retained by the author(s) and / or other copyright owners and it is a condition of accessing these publications that users recognise and abide by the legal requirements associated with these rights.

Take down policy

The Research Portal is Queen's institutional repository that provides access to Queen's research output. Every effort has been made to ensure that content in the Research Portal does not infringe any person's rights, or applicable UK laws. If you discover content in the Research Portal that you believe breaches copyright or violates any law, please contact openaccess@qub.ac.uk. 


\title{
Evaluation of crystallographic strain, rotation and defects in functional oxides by the moiré effect in scanning transmission electron microscopy
}

\author{
AB Naden, ${ }^{1,2}$ KJ O’Shea ${ }^{2}$ and DA MacLaren ${ }^{2}$ \\ ${ }^{1}$ Present Address: School of Mathematics and Physics, Queen's University Belfast, Belfast, BT7 1NN, UK \\ ${ }^{2}$ SUPA, School of Physics and Astronomy, University of Glasgow, Glasgow, G12 8QQ, UK \\ E-mail: abnaden@physics.org, damaclaren@physics.org
}

\begin{abstract}
Moiré patterns in scanning transmission electron microscopy (STEM) images of epitaxial perovskite oxides are used to assess strain and defect densities over fields of view extending over several hundred nanometers. The patterns arise from the geometric overlap of the rastered STEM electron beam and the samples' crystal periodicities and we explore the emergence and application of these moiré fringes for rapid strain analysis. Using the epitaxial functional oxide perovskites $\mathrm{BiFeO}_{3}$ and $\mathrm{Pr}_{1-x} \mathrm{Ca}_{x} \mathrm{MnO}_{3}$, we discuss the impact of large degrees of strain on the quantification of STEM moiré patterns, identify defects in the fringe patterns and quantify strain and lattice rotation. Such a wide-area analysis of crystallographic strain and defects is crucial for developing structure-function relations of functional oxides and we find the STEM moiré technique to be an attractive means of structural assessment that can be readily applied to low dose studies of damage sensitive crystalline materials.
\end{abstract}

Keywords: functional oxides, moiré, strain, scanning transmission electron microscopy 


\section{Introduction}

Crystallographic strain manifests in a wide range of materials and can have a profound effect on a material's functionality. It can be tuned, for example, by atomic-scale features such as doping [1] or an epitaxiallymatched interface [2] yet can manifest over micron length-scales, and this wide range of dimensions makes accurate characterisation problematic. A number of different techniques are available for the precise assessment of strain but they typically offer either relatively poor spatial resolution of tens of nanometres or are limited to small fields of view [3]. Nanobeam electron diffraction, for example, can be used to accurately assess strain on the nanoscale, but can be slow to implement point-by-point across a large field of view without additional hardware. [4],[5] Scanning transmission electron microscopy (STEM) is particularly versatile for nanoscale analysis, a key advantage being the atomic-scale spatial resolution now afforded by aberration-corrected instruments, which enables the direct visualisation of atomic columns and, therefore, direct quantification of deviations from perfect crystal periodicity. However, practical constraints on data set sizes and acquisition times generally limit the field of view for such analysis to around $25 \mathrm{~nm}$, which may be too small to assess low defect densities. A series of recent studies has demonstrated that the STEM-moiré technique can address the problem of simple large area strain measurement [6-16]. The pixel-wise nature of STEM imaging means that if the atomic columns (crystallographic planes) of a crystalline specimen are under-sampled, a moiré pattern is formed, analogous to the aliasing effect observed in image processing [17], fluorescence microscopy [18] and, indeed, in conventional TEM $[19,20]$. In each of these cases, a moiré pattern arises from the overlap of two periodic patterns, typically a reference pattern and a sample, to produce a homothetic image that appears as a magnified reproduction of the sample lattice, including its imperfections, thereby offering excellent sensitivity to variations in lattice spacing over fields of view inaccessible to more popular methods. One advantage is that the STEM scan itself forms one of the two periodic patterns required for moiré fringe formation, so that a separate reference lattice is not required (once the STEM magnification has been calibrated). Another advantage is that STEM moiré uses much lower electron doses per unit area than atomically-resolved STEM, which is particularly beneficial for beam sensitive materials. In the past few years, STEM-moiré using high angle annular dark field (HAADF) imaging has been applied to study strain and dislocations in Si-based semiconductor devices $[8,10,13]$ and epitaxial oxide systems $[15,21]$, but the technique has yet to gain wide-spread usage. 
In this report, we first summarise the mathematical description of the STEM moire effect and use geometric phase analysis (GPA) [22] to assess spatial variations in moiré fringe separations. We extend a recent Fourier-based description [16] to explore two-dimensional moiré patterns in more detail and to discuss cases where the STEM scan spacing is a fraction of the crystal unit cell size; and we show the approach to simplify the 'traditional' (but less insightful) equations that have been used elsewhere $[8,15]$. We then apply our STEM moiré analysis to two functional epitaxial oxides, $\mathrm{BiFeO}_{3}(\mathrm{BFO})$ and $\mathrm{Pr}_{0.48} \mathrm{Ca}_{0.52} \mathrm{MnO}_{3}(\mathrm{PCMO}$ ), which were chosen to illustrate cases of inhomogenous strain and lattice rotation.

Transition metal oxide films have been found to possess a variety of functionalities including ferroelectricity, ferromagnetism, multiferroicity, superconductivity [2] and resistive switching [23], characteristics that are often sensitive to minor structural changes in bond lengths and angles [24]. Such changes can be induced in thin films by the introduction of dopant atoms [1, 25], control of oxygen stoichiometry [24] or epitaxial strain during film growth [2]. BFO is a promising multiferroic due to the fact that it is both ferroelectric and antiferromagnetic at room temperature [26] leading to potential applications in spintronics [27] and ferroelectric memories [28]. Here, a $\mathrm{DyScO}_{3} / \mathrm{SrRuO}_{3} / \mathrm{BiFeO}_{3}(\mathrm{DSO} / \mathrm{SRO} / \mathrm{BFO})$ sample is presented and we quantify strain over fields of view of $\sim 200 \mathrm{~nm}$ and identify a low density of dislocations. Comparison of values of the lattice spacing measured from STEM moiré and high-resolution STEM reveals the excellent degree of accuracy of the former, with an agreement within 2-3 pm between the two techniques. $\operatorname{Pr}_{1-\mathrm{x}} \mathrm{Ca}_{\mathrm{x}} \mathrm{MnO}_{3}$, on the other hand, has attracted significant interest for applications such as resistive random access memory $[29,30]$. In the $\mathrm{SrTiO}_{3} / \mathrm{Pr}_{0.48} \mathrm{Ca}_{0.52} \mathrm{MnO}_{3}$ (STO/PCMO) system, we measure lattice rotations caused by cracks that relieve a large degree of inhomogeneous strain [31], again demonstrating the efficacy of STEM moiré over large fields of view. This ability to measure small lattice rotations has not been covered in detail in previous STEM moiré work but, alongside an analysis of defects and the deformations of crystal lattices over large areas, could find application in studies of a number of functional materials.

\section{Experimental}

Samples were fabricated elsewhere by pulsed laser deposition of SRO and BFO on a (110)o-oriented DSO substrate [32] and PCMO on an STO substrate [31], with details provided in the preceding references. Crosssections for STEM were prepared using focused ion beam milling in an FEI Nova DualBeam instrument equipped with an Omniprobe micromanipulator for in-situ lift out [33]. Milling was performed using 30keV 
$\mathrm{Ga}^{+}$beam for bulk milling and a $5 \mathrm{keV}$ beam for a final polish. We then employed HAADF STEM due to its strong image contrast [34], using JEOL annular dark field detectors with inner and outer collection angles of 68 and $230 \mathrm{mrad}$, respectively, on a probe-corrected JEOL ARM200cF instrument operated at $200 \mathrm{kV}$. STEM images were acquired using a Gatan DigiScan II system and analysed using Gatan Digital Micrograph and Matlab. Geometric phase analysis was performed using our own Matlab code that followed the method described elsewhere $[22,35]$. To assess the impact of scanning spacing, pixel acquisition (dwell) time and scan orientation on STEM moiré images, data were acquired with a fixed pixel resolution of $512 \times 512$ pixels, nominal magnifications ranging from 0.6 to 20 million times (Mx) (ranging from 0.619 to $0.019 \mathrm{~nm} / \mathrm{pix}$, respectively), pixel dwell times ranging in integer multiples from $38.9 \mu$ s (50 $\mathrm{Hz}$ mains synchronisation) to $155.6 \mu$ s and at orthogonal scan orientations of 0 and 90 . Variations in dwell time additionally provided a simple means to assess time-dependent distortions such as sample/stage drift.

\section{Moiré fringe formation \& analysis procedure}

In STEM moiré, fringe patterns are formed as the result of under-sampling the periodic crystallographic planes of a specimen with the periodic scan of the STEM probe. Two dimensional moiré patterns can arise when aliasing occurs along two orthogonal axes - the fast and slow scan axes - although in some cases we find flyback errors [36], instabilities and sample drift [37] can diminish the visibility of fringes and alter their separation along the slow scan axis. STEM images can then be considered as the sampling of a projected crystal potential (the atomic columns) with a scanned atomic-scale probe and thus the same requirements of atomically-resolved, high resolution STEM (HR-STEM), namely microscope stability and resolution, also apply to STEM moiré. In the case of the specimen, the projected crystal potential derives from the convolution of a two-dimensional lattice of delta-functions with a basis of atomic potentials associated with each lattice site, giving a two-dimensional periodic function, here termed $G_{s}(\mathrm{r})$. This may be further convolved with the probe's intensity distribution, although for the narrow probes required for atomic-resolution STEM, the factor can be ignored. Similarly, the scan pattern is a square lattice of probe (or pixel) positions, here termed $G_{r}(\mathrm{r})$ (where the subscript ' $r$ ' refers to the 'reference' lattice). To obtain the sampling effect, the resulting STEM image (which may contain moiré terms) is then given by $G_{m}(r)=G_{r}(r) G_{s}(r)$, the multiplication of the two arrays. An insightful way to determine the frequency components that contribute to moire fringes is then to use a reciprocal space representation, as discussed comprehensively elsewhere [38, 39]. Using the convolution 
theorem, the multiplication of the specimen and reference arrays in real space is represented by the convolution of their respective Fourier transforms in reciprocal space. Both $G_{s}(r)$ and $G_{s}(r)$ have Fourier transforms that are arrays of spots. The convolution of these two spot patterns is therefore generated by replicating the specimen reciprocal lattice at each reciprocal lattice spot of the scan function. The process is outlined in figure 1, which presents schematics of (a) 1-dimensional (1d) and (b) 2-dimensional (2d) moiré patterns produced from the overlap of arrays of lines and spots, respectively, with both the spatial frequency and the rotation angle of the specimen lattice (in blue) altered with respect to the reference lattice (in red). The plot of spatial frequencies in Figure 1(c) provides an intuitive overview of moiré pattern formation. Here, both the reference and specimen $2 \mathrm{~d}$ lattices produce square arrays of spots, with a mutual rotation angle $\alpha$ and a spacing that scales with the inverse of the real-space lattice periodicities, here termed the lattice frequencies. Moiré fringes can be predicted on the basis of vector addition of the reference and specimen lattice frequencies, $f_{r}$ and $f_{s}$, respectively: for example, the moiré fringes in figure 1(a) arise from $\left|f_{r}-f_{s}\right|$, producing a pair of spots a distance $\left|f_{r}-f_{s}\right|$ from the origin in figure 1(c); they are at angle $\theta$ to the $f_{x}$-axis, consistent with the angles in the upper panels, which are measured with respect to a line lying perpendicular to the moiré fringes. Other, higher-order moiré fringes are produced by other integer combinations of frequency vectors or, alternatively, by replicating the sample's array of blue spots around each of the reference crosses, in the manner of convolutions. (In practice, the intensity of the higher-order features is insignificant, such that only the lowest-order moiré fringes are readily observed [38].) The visibility of moiré fringes depends on a number of factors, but they typically need to have a maximum wavelength of half the scan size and a minimum wavelength of around four crystal unit cells to be clearly discerned from other intensity variations: the resulting annular region of frequency space is indicated by the dashed circle in figure 1(c). Clearly, the positions of potential moiré spots in figure 1(c) depend on both the angle between the frequency vectors and their relative lengths, with STEM scans that don't give rise to strong moiré fringes producing $\left|f_{r}-f_{s}\right|$ spots outside of the marked circle.

For the one-dimensional fringes indexed in figure 1(c), application of the cosine rule to the triangle of $f_{r}, f_{s}$ and $f_{m}$ demonstrates that the above pictorial approach is equivalent to the often-quoted formulae for moiré fringes arising from the overlap of two closely-spaced frequencies $[15,20]$, which we here write in terms of reciprocal lengths (i.e. frequencies), and solve for the specimen frequency, which is generally the unknown 
parameter in an experiment. If the lattices are rotated by an angle, $\alpha$, with respect to one another, then fringes lie at an angle $\theta$ [see figure 1(a), (b)] and the frequency of the specimen lattice can be determined using:

$f_{s}(r)=\sqrt{f_{r}^{2}+f_{m}(r)^{2} \pm 2 f_{r} f_{m}(r) \cos \theta(r)}$,

and

$\cos \alpha(r)=f_{s}^{2}(r)+f_{r}^{2}-f_{m}(r)^{2} / 2 f_{r} f_{s}(r)=\frac{f_{r}}{f_{s}} \pm \frac{f_{m}}{f_{s}} \cos \theta(r)$,

where a positive sign is taken in Eq. (1) for $f_{s}>f_{r}$ and a negative sign for $f_{s}<f_{r}, \theta$ is measured as the acute angle with the positive $f_{x}$-axis in figure 1 (c) (which is the scan direction) and $f_{s}=1 / d_{s}$ for a sample lattice spacing of $d_{s}$, etc. Note that we have explicitly written both $f_{s}$ and $\theta$ as functions of position, since in real specimens, the spatial variations in lattice strain and angle are of greatest interest. This $1 \mathrm{~d}$ analysis is useful because it is often the case that minor perturbations in the STEM scan, including fly-back errors [37, 40], reduce the expected $2 \mathrm{~d}$ moiré patterns for a well-ordered crystal specimen to a $1 \mathrm{~d}$ set of fringes as discussed in section 1 of the Supplementary Material. An important consequence of the above equations is that it is necessary to first know whether $d_{s}$ is greater or less than $d_{r}$ since using the wrong form of Eq. 1 (with respect to the plus/minus sign) would lead to significantly different values of $d_{s}$. However, in most cases it ought to be sufficient to collect a high resolution, lattice resolved image to determine $d_{s}$ locally. This is not a major hindrance, since, for STEM moiré fringes to form, the electron probe must be small enough to resolve atomicscale structure. Despite this sensitivity, the STEM moiré technique cannot ultimately afford the same spatial resolution as HR-STEM because the imaging conditions will generally not satisfy the Nyquist sampling rate and so will give rise to aliasing - a consequence of which is the moiré fringes of interest here. Typically, a number of lattice spacings are required to effectively average out into fringes in addition to the processing constraints which are discussed in section 2 of the Supplementary Material. The spatial resolution of STEM moiré depends on the proximity of $f_{r}$ and $f_{s}$, the spot size and beam stability [37] and is typically of the order of several nanometers, scaling with the wavelength of the moiré fringe. Typically, at least a quarter wavelength - for example, maximum to minimum in fringe intensity - would be necessary to accurately determine the local strain, so it may be necessary to adjust the scan conditions to maximize the number of fringes in the field of view. Although there is no hard and fast rule, we estimate that, as a very rough guide, maintaining $f_{r} \sim f_{s} \pm 0.1 f_{s}$ will provide useful STEM moiré patterns. The technique's main advantage, however, is its ability to assess strain over large fields of up view of hundreds of nanometers, which would 
require prohibitively long acquisition times in HR-STEM, making STEM moiré a highly useful complementary technique.

Equations (1) and (2) are strictly only appropriate for situations where $f_{s} \approx f_{r}$, which can be considered 'first order' moiré effects; however, at low magnifications, moiré fringes and homothetic crystal images can also be formed when the STEM probe only samples every $n^{\text {th }}$ unit cell [7]. The resulting 'higher order' moiré effects are easily accommodated within the methodology outlined in figure 1 , by replacing the requirement for a small $\left|f_{r}-f_{s}\right|$ with a small $\left|n f_{r}-f_{s}\right|$ where $n$ is an integer and several vectors are added to produce a moire spot close to the origin; the resulting frequency plots become more crowded because the number of reciprocal reference points (the red crosses) in figure 1 increases, as does the number of clusters of spots, but it need not increase the number of visible moiré frequencies within the dashed circle. Practically, the lowest magnification at which moiré fringes are observed will depend on the resolution of probe positioning across a large field of view. In a similar manner, we find that situations with small $\left|f_{r}-m f_{s}\right|$ where $m$ is an integer - i.e. where the STEM probe makes $m$ samples per unit cell - can also give rise to weak moirélike phenomena that here we refer to as 'fractional moiré' effects. In these cases, the Nyquist sampling rate may or may not be achieved with regards to the unit cell periodicity (or the maximum spatial frequency, which will be higher for non-primitive cells) and isolating the moiré signals can become problematic [41] as they tend to appear as $m$ overlapping, phase-shifted 'envelope functions' that modulate the image data. Figure 2 provides an overview of typical moiré signals, calculated for a (100)-oriented $\mathrm{BFO}$ crystal at a range of magnifications (with $\alpha=0^{\circ}$ and with magnifications appropriate to our JEOL ARM instrument). Each plot shows, in black, the atomic potential along a (001) direction and through the A-site of the perovskite cell; the coloured plot then shows the calculated pixel intensity. The top trace simulates a line-scan through an image collected with a magnification of $0.5 \mathrm{Mx}(0.5$ million times $)$, for which $f_{r}=1.3466 \mathrm{~nm}^{-1}, f_{s}=2.524 \mathrm{~nm}^{-1}$ and hence which has $2 f_{r} \approx f_{s}$, here termed a 'higher order' moiré effect with only every second crystal cell sampled. This produces an identical moiré periodicity to the 'first order' effect recorded at a magnification of $1.0 \mathrm{Mx}$, which is shown in the second trace, where $f_{r} \approx 0.9 f_{s}$ and every cell is sampled. STEM images and their Fourier transforms of a real image collected under these conditions are presented in section 2 of the Supplementary Material. At $1 \mathrm{Mx}$ magnification, the unit cells are not directly resolved and the low frequencies of the moiré fringes can be observed near the origin of the FFT. This first order case represents the most common acquisition settings for STEM moiré and fringes typically have the strongest contrast. At a 
magnification of $2 \mathrm{Mx}$ (figure 2, third trace), the sampling frequency is approximately double the crystal cell periodicity (i.e. two STEM probe positions per unit cell) producing the 'fractional moiré effect'. Moiré peaks are again visible in the FFT and a line-section of the image clearly shows two modulating envelope functions, both of which have the same period as that of the first-order effect, as illustrated. The Fourier transform now shows a moiré peak close to the origin but also a lattice peak and both frequencies can be extracted by Fourier filtering (see section 2 of the supplementary information). Finally, at a magnification of $3 \mathrm{Mx}$ (figure 2, bottom trace), the intensity modulation is reduced and although the FFT clearly resolves peaks that correspond to the unit cell periodicity, the expected moiré peaks close to the origin are not resolved. The line-profile through the data now contains three envelope functions that cannot be extracted by conventional FFT techniques (a more detailed discussion is given in the literature [41]) but a Fourier-filtered image clearly shows that the lattice periodicity is modulated by moiré envelope functions as shown. In practice, we did not readily observe moiré effects for $f_{r}>3 f_{s}$.

Once a STEM moiré pattern is formed, an appropriate method for measuring the fringe separation is required. The analysis set out above considered lattices of uniform spatial frequency but the real value of the STEM moiré technique is in analysing samples where the spatial frequencies change with position. The vector schematic of figure 1(c) indicates that changes in either $\alpha$ or $f_{s}$ (provided $\alpha$ is non-zero) will produce a change in moiré fringe orientation, to produce curved fringes. This can complicate analysis since separating the two contributions is difficult and $\theta$ is not always known to a high degree of accuracy. These aspects necessitate a local measure of the fringe separation. For this, Geometric Phase Analysis (GPA) is particularly well suited and gives accurate continuous geometric phase distributions, from which the local fringe and hence lattice spacings can be determined. It is typically applied to high magnification images where atomic positions and thus the lattice spacings can be determined directly; here, we apply it to the moiré fringes themselves. A full discussion of GPA can be found elsewhere [22, 35, 42]. We find that, based on calculated moiré images, the error in the GPA measurement of $d_{s}$ is of the order of $10^{-4} \mathrm{~nm}$ and the error in $\theta$ is approximately $0.3^{\circ}$. This provides an upper limit to the anticipated precision since aspects such as scan distortion and stage drift will inevitably degrade the experimental precision. This illustrates the excellent sub-picometre and sub-degree sensitivity of the measurements, similar to a previous report that determined the experimental accuracy to be around $1 \mathrm{pm}$ for lattice-resolved HR-STEM data [43]. The fundamental limits on the precision and spatial resolution of measurements of ideal STEM moiré patterns by GPA lie in the size and type of window used for 
the Fourier transform discussed in detail elsewhere [16, 43], the numerical precision in the calculation of the Fourier transform, the fringe separation (the spatial resolution scales with the fringe wavelength but there must be a separation of at least 1 pixel) and the accuracy in the microscope calibration of $f_{r}$.

\section{Applications of STEM moiré}

\section{1 $\mathrm{BiFeO}_{3}$}

In this section we discuss $2 \mathrm{D}$ moiré images of a $\mathrm{DSO} / \mathrm{SRO} / \mathrm{BFO}$ system in order to assess strain over a large field of view and we compare the derived lattice spacings to those measured by HR-STEM (see section 3 of the Supplementary Material for details) to verify the accuracy of the moiré measurements. To minimise the potentially convoluted effects of fly-back error and stage drift (which is also time-dependent), images were collected with the fast scan axis at two orthogonal orientations and from the same region, then strain was quantified along the fast scan axes. Other approaches to mitigating fly-back error and drift include the collection of a series of images with low acquisition times and correcting for distortion in post processing [44] and 'revolving STEM' where a succession of images is recorded with rotating scan angle [45].

Figure 3a shows one of the 'raw' HAADF first order moiré images, oriented with the fast scan direction along the $[001]_{C}$ DSO axis, i.e. the $[110]_{0}$ axis, where the ' $\mathrm{C}$ ' and ' $\mathrm{O}$ ' subscripts refer to pseudocubic and orthorhombic, respectively.[46] The horizontal axis is labelled as $[100]_{\mathrm{C}}$ for reference. The SRO layer runs down the centre of the image and appears darkest because the intensity of atomic columns varies with the square of the atomic number $(\mathrm{Z})$. Fringe contrast in the SRO layer is further diminished because the $\mathrm{A}$-site $(\mathrm{Sr})$ and $\mathrm{B}$-site $(\mathrm{Ru})$ atoms are relatively close in $\mathrm{Z}$, particularly in comparison to the larger Z-differences in both the DSO and BFO layers. A number of further images that show better the contrast variations and how they vary with acquisition parameters are presented in section S3 of the Supplementary Material. Figure $3 b$ shows a Fourier filtered image, selecting only the moiré frequencies in figure $3 a$ and revealing more clearly the 2D moiré fringe pattern, with reduced fringe visibility within the central SRO layer. Note in particular that the spots align along different angles in the DSO and BFO layers, consistent with the change in lattice parameter, and that there is no strong bending, suggesting a relatively uniform lattice spacing in each of the two layers. It is also worth noting that in both DSO and BFO, the A-site atom (Dy and Bi, respectively) has higher $\mathrm{Z}$ and so dominates the HAADF-STEM images whilst in SRO the B-site atom (Ru) dominates STEM image contrast, leading to the expectation of apparent antiphase boundaries in the moiré 
fringe patterns along the two interfaces, which is clearly observed in the bottom portion of figure $3 \mathrm{~b}$. Figures $3 \mathrm{c}$ and $3 \mathrm{~d}$ show strain maps along the $[100]_{\mathrm{C}}$ and $[001]_{\mathrm{C}}$ axes, respectively, and are derived from the fast scan axes of the raw moiré images. The strain is calibrated with respect to the measured lattice spacing of bulk rhombohedral BFO to the right of the image, listed in table 1 along with the values for the other materials discussed in this system. Strain is defined according to $s[\%]=\left(\left(a_{r}-a_{s}\right) / a_{r}\right) \times 100$ where $a_{r}$ is the reference or 'bulk' lattice spacing and $a_{s}$ is the measured specimen value. The $[100]_{\mathrm{C}}$ fringes in figure $3 \mathrm{a}$ are oriented differently within the DSO and BFO layers, so the images were processed in two parts and a mask placed over the SRO layer, which is shown in black in figure 3c.

We begin the analysis by considering the accuracy of the moiré measurements. Table 2 contains measured mean values of lattice spacing derived from the STEM moiré data in figure 3 and equivalent values measured from HR-STEM. From both strain maps in figure 3, there is an excellent correlation to within $2-3$ pm of the HR-STEM measurements, suggesting a high degree of accuracy. However, the strain maps also suggest a degree of spatial inhomogeneity away from the interfaces that is not apparent across the narrow fields of view used for HR-STEM. Filtering of Fourier transforms inevitably introduces unphysical oscillations and edge effects into the maps produced by GPA analysis [22, 35, 42], to which we attribute the red regions at the edges of figure $3 \mathrm{c}$. Away from the edges, the difference between the blue/green and yellow regions corresponds to a $0.16 \%$ or $0.6 \mathrm{pm}$ variation of the in-plane lattice constant across a $\sim 150 \mathrm{~nm}$ region. This explains the increase in the error quoted for moire measurements in comparison to HR-STEM in table 2 and we attribute the apparent inhomogeneity to a low level of sample drift and/or beam instability, as discussed elsewhere [16]. Along [100]C, strain values of $\sim 1.6$ to $1.9 \%$ in the DSO layer and $\sim 2.1$ to $2.3 \%$ in $\mathrm{BFO}$ are measured with respect to their bulk values (note that the strain maps in figure 3 are scaled with respect to the bulk value of BFO for representative purposes).

Turning to the $[001]_{\mathrm{C}}$ fringes in figure $3 \mathrm{~d}$, apparent defects in the moiré fringes at the SRO/BFO interface can be identified and manifest as the three dark blue streaks that we attribute to a limitation in moiré analysis that has not been described previously: it is important because it is tempting to attribute the streaks to interfacial strain-relief dislocations, which is not the case. Defects such as dislocations are known to play a crucial role in the functional properties of ferroelectric materials where they can act as pinning sites for ferroelectric domain walls [47] and it is essential that they can be assessed accurately. 
The streak features can be seen in more detail by Fourier filtering the fringe patterns as shown in figure 4 . Figures $4 \mathrm{a}$ and $4 \mathrm{~b}$ show FFTs corresponding to the strain maps in figures $3 \mathrm{c}$ and $3 \mathrm{~d}$, respectively. The annotations show the reflections corresponding to the fringes that can be seen in the original moiré image (figure 3a) and the red circles depict the size and location of the windows applied. A spot splitting can be seen in both directions; along $[100]_{\mathrm{C}}$, this arises due to the change in orientation of the fringes in the DSO and BFO layers whereas along $[001]_{C}$ it arises from the different spacing in these layers. The inverse FFTs (IFFTs) of figures $4 \mathrm{a}$ and $\mathrm{b}$ were taken using only the regions defined by the windows and are shown in figures $4 \mathrm{c}$ and 4d. Along $[001]_{C}$ (figure $4 \mathrm{c}$ ), the fringes appear as vertical white lines whereas they are horizontal along [100]c (figure 4d). The red arrows mark the termination of the filtered moiré fringes at both the DSO/SRO and SRO/BFO interfaces, which appear at the same points in both images.

The moiré fringes in figure $3 \mathrm{a}$ are angled due to a small tilt of the underlying crystal lattices with respect to the scan pattern; and as the angle of the moiré fringes depends on the crystal lattice spacing, the fringes appear at different angles in each of the three materials (note that the fringe angles are not homothetic to the angle of the underlying crystal lattice). Thus at the interfaces, where the moire spot patterns meet, there are necessarily discontinuities in the fringes that are also not homothetic to the underlying lattice. The discontinuities appear as crystallographic dislocations in the resulting strain maps, even though high resolution STEM imaging does not indicate their presence. The effect is illustrated in more detail in section S4 of the supporting information. This is therefore an important distinction that must be considered when processing STEM moiré patterns of heteroepitaxial systems. The ability to rapidly assess crystallographic strain via moiré fringe patterns should find wide applicability yet extending the technique to study of defects such as dislocations [8] evidently requires care. We therefore recommend that moiré imaging is used to locate potential defect sites that are then analysed directly with higher resolution.

Returning to figure 3, near the $\mathrm{SRO} / \mathrm{BFO}$ interface, the strain in $\mathrm{BFO}$ varies rapidly but reaches a maximum magnitude of $s \sim-1.5 \%$ and then apparently relaxes within the streaked region up to $\sim 25 \mathrm{~nm}$ from the interface. Beyond this, a more uniform relaxation takes over and a value of $s \sim-0.5 \%$ is attained $\sim 50 \mathrm{~nm}$ from the interface, toward the far right hand side of the map in figure $3 \mathrm{~d}$. Thus, an inhomogeneous strain relaxation is present. These strain values agree with previously reported values of $c$-axis values of $\sim-0.8 \%$ to $\sim-0.1 \%$ for BFO grown on DSO $[48,49]$ and $\sim-1.8 \%$ on STO [49] as measured by X-ray diffraction. 
Coupled with the $[100]_{\mathrm{C}}$ measurements above, this corresponds to a tetragonal distortion of BFO $(c / a \sim 1.03)$. The corresponding values for the lattice spacings within BFO suggest a unit cell volume of $59.7 \AA^{3}$ in the 'relaxed' region compared to the bulk value of $62.2 \AA^{3}$, a reduction of $\sim 4 \%$. Similar values are obtained from the HR-STEM data with a volume of $59.1 \AA^{3}$ in the same region. Within the DSO layer, the strain with respect to the DSO bulk value peaks at around $0.8 \%$ near the SRO interface (within approximately $20 \mathrm{~nm}$ ) and decreases to $\sim 0.05 \%$ at the far left hand side of figure $3 \mathrm{~d}$, i.e. the lattice spacing approaches the bulk value. This is curious since it might be expected that the single crystal DSO substrate would impose its crystallography on the much thinner SRO and BFO overlayers rather than vice-versa. However, it has been noted previously that an important aspect of epitaxial growth is elastic coupling between the growing film and the bulk substrate $[50,51]$ and that the Young's modulus of DSO is highly anisotropic and lowest along $[100]_{\mathrm{C}},[52]$ a result consistent with the increase in strain along $[100]_{\mathrm{C}}$ observed here. Furthermore, similar behaviour has previously been observed in very similar materials systems using X-ray diffraction and it was shown that the ferroelectric domain structure was linked to this strain $[48,53]$. STEM moiré can therefore afford an important insight into strain accommodation in both the functional film and its substrate that is crucial for understanding the origin of the functional properties of epitaxial thin films [53]. There do not appear to be any previous reports discussing the strain relaxation in this particular materials system with the high spatial resolution but large field of view discussed here, however relaxation over film thicknesses up to around $200 \mathrm{~nm}$ has been reported for similar systems employing differing substrates [54].

\subsection{Lattice Rotation in Strained, Cracked $\mathrm{Pr}_{0.48} \mathrm{Ca}_{0.52} \mathrm{MnO}_{3}$}

In this final section, we analyse data with low fringe visibility and apply the developed approach to assess variations in the rotation of otherwise uniform lattices. Figure 5a shows a predominantly 1D moiré image (collected with a dwell time of $38.9 \mu$ s where fringe visibility along the fast scan direction is low) of an STO/PCMO system where the value of $d_{r}$ was close to half the unit cell dimension of PCMO and STO, resulting in a fractional moiré effect of the type illustrated in figure 2. A large crack can be seen along the centre of the image, formed during film growth to relax a large degree of stress, as investigated in detail in a previous report [31]. That report discussed the complex relaxation behaviour in this system that plays an important role in determining the electrical properties of the film. For our purposes, the curvature of the 
fringes in figure 5a arises primarily due to inhomogeneous rotations of the specimen lattices on either side of the crack and propagating into the substrate, as confirmed by the atomic resolution studies discussed in section 3 of the Supplementary Material. Since strain was relieved by the crack, we assume a fixed value of $d_{s}$ measured from the HR-STEM data (see Supplementary Material) and calculate the variation in lattice rotation, $\theta$, using Equation 3.

As illustrated by the very weak reflections in the FFT in figure 5b, the fringe visibility is low. The yellow annotation denotes the expected location of the fringe frequencies in the FFT, but they are too weak to be extracted reliably. However, the high frequency unit cell $d_{s}(100)$ fringes can be seen and are shown by the red annotations in the FFT in figure 5b. The amplitude of these fringes is modulated by a moire envelope, as can be seen in the IFFT in figure $5 \mathrm{c}$ and the corresponding profile in figure $5 \mathrm{e}$. In order to recover the low frequency envelope we found that down-sampling by $2 \times 2$ nearest neighbour interpolation $(2 \times 2$ binning $)$ was most effective. A downsampled version of figure $5 \mathrm{a}$ is shown in figure $5 \mathrm{~d}$.

Figure 6a shows a moiré fringe image produced by Fourier filtering and down-sampling the raw image in figure 5a along the $[100]_{\mathrm{C}}$ direction. $|\theta|$ is shown in figure $6 \mathrm{~b}$ for representative purposes. It was necessary to process this image in separate halves by applying appropriate masks to the images, shown by the black regions, in order to avoid errors in the GPA procedure. The dashed black lines indicate the location of the STO/PCMO interface. A constant value of $d_{s}=0.380 \mathrm{~nm}$ (the bulk pseudocubic value [55] of $\operatorname{Pr}_{0.5} \mathrm{Ca}_{0.5} \mathrm{MnO}_{3}$ ) was set based on measurements from the HR-STEM data since the relatively small variations in lattice spacings of a couple of pm resulted in a difference of $\sim 0.03^{\circ}$ in the derived values of $\theta$, much less than the anticipated maximum accuracy of $0.3^{\circ}$ discussed in section 3 .

Above the crack, in the upper half of the image, there is a clockwise rotation and an anticlockwise one below. It can be seen from figure $6 \mathrm{~b}$ that $\theta$ is maximised in the immediate vicinity of the base of the crack at the STO/PCMO interface and that it then gradually decreases as function of distance from the crack. Within the PCMO layer, $\theta$ decreases from a maximum value of $\sim 2.2^{\circ}$ at the base of the crack to $\sim 0.5^{\circ}$ toward the upper edge of the image, in good agreement with the value of $\sim 3^{\circ}$ measured near the base of the crack by HRSTEM. This therefore illustrates that the STO/PCMO film stack adopts a large and opposing rotation either side of the crack, gradually relaxing toward $0^{\circ}$ with increasing distance from the crack. This suggests a small amount of strain relaxation and/or incorporation of dislocations however we do not observe any dislocations 
in either STEM moiré or HR-STEM. This measurement of $\theta$ by STEM moiré demonstrates the versatility of the technique, enabling the characterisation of crystallographic rotation over large fields of view which may be of particular interest for the investigation of features such as ferroelastic twin boundaries which can exhibit practically negligible differences in lattice spacing but with small crystallographic rotations [56].

\section{Conclusions}

In summary, we have presented an overview of the formation and analysis of the STEM moiré effect, including a mathematical description that links fringe formation to accurate analysis for a variety of image acquisition magnifications, including both 'higher order' and 'fractional' moiré effects. Although such effects have been described in other image formation processes, their significance to STEM moiré has not been discussed in detail previously. We find that application of geometric phase analysis to STEM moiré analysis is ideal for an assessment of inhomogenous strain and lattice rotation over wide areas. This combined analysis will prove invaluable for optimised acquisition of future datasets.

We have illustrated the versatility - and some limitations - of STEM moiré by considering two technologically relevant functional oxide systems, identifying dislocations in the moiré fringes and quantifying strain, tetragonality and crystallographic rotation over large fields of view up to $\sim 200 \mathrm{~nm}$. Additionally, we have demonstrated the application of the technique to measurement of inhomogeneous lattice rotation to an anticipated accuracy of $\sim 0.3^{\circ}$. A crucial advantage of this technique is the ability to garner this large amount of information from a small dataset with relative ease and we therefore envisage that this will become an excellent complementary technique to existing STEM analyses. Our approach is well suited for the analysis of strain in technologically relevant materials including - but not limited to - the functional oxides discussed here, and could lead to a greater understanding of the links between strain evolution in a thin film and the material's functional properties, which is central to the field of materials science.

\section{Acknowledgements}

ABN gratefully acknowledges the Engineering and Physical Sciences Research Council (EPSRC) of the UK for provision of a doctoral training grant through $\mathrm{EP} / \mathrm{J} 500434 / 1$. KJO acknowledges funding from the European Union Council under the $7^{\text {th }}$ Framework Program (FP7) grant no. 246102 IFOX. DAM is grateful 
for a fellowship from the EPSRC through contract EP/I00419X/1. The authors also wish to thank I. LindforsVrejoiu (currently at II. Physikalisches Institut, Universität zu Köln, Köln, Germany) and A. Herpers and R. Dittmann (Research Center Jülich, Peter Grünberg Institute, Electronic Materials, Jülich, Germany) for fabricating and providing the samples used in this study.

\section{Supplementary material}

See supplementary material for discussions of the effects of magnification and pixel dwell time on the observed moiré patterns and for details of the HR-STEM analyses complementing section 4. 
Tables

Table 1. Bulk (pseudo)cubic values for the materials in the BFO system, the original references are listed in the table.

\begin{tabular}{cccc} 
Material & $a_{c}[\mathrm{~nm}]$ & $b_{c}=c_{c}[\mathrm{~nm}]$ & Reference \\
\hline DSO & 0.3952 & 0.3946 & {$[57]$} \\
SRO & 0.3923 & 0.3923 & {$[58]$} \\
BFO & 0.3962 & 0.3962 & {$[59]$} \\
\hline
\end{tabular}

Table 2. Lattice spacings as measured from the fast scan axes of the HR-STEM images in the Supplementary Material and the moire measurements in section 4 . The values quoted are mean \pm standard deviation measured across the layers within the GPA maps. The values quoted for the moiré measurements of the BFO $d_{s}$ [001] spacings correspond to the 'relaxed' regions of the film $\sim 50 \mathrm{~nm}$ from the interface.

\begin{tabular}{cccc}
\hline Technique & Material & $d_{s}[100][\mathrm{nm}]$ & $d_{s}[001][\mathrm{nm}]$ \\
\hline HR-STEM & DSO & $0.3864 \pm 0.0001$ & $0.3924 \pm 0.0001$ \\
HR-STEM & BFO & $0.3845 \pm 0.0008$ & $0.4000 \pm 0.0007(\max )$ \\
& & & $0.3960 \pm 0.0008(\min )$ \\
Moiré & DSO & $0.3882 \pm 0.0002$ & $0.3945 \pm 0.0002$ \\
Moiré & BFO & $0.3876 \pm 0.0002$ & $0.3972 \pm 0.0002$ \\
\hline
\end{tabular}




\section{References}

[1] Guo H, Dong S, Rack P D, Budai J D, Beekman C, Gai Z, Siemons W, Gonzalez C M, Timilsina R, Wong A T, Herklotz A, Snijders P C, Dagotto E and Ward T Z 2015 Phys.Rev.Lett. 114256801

[2] Martin L W, Chu Y H and Ramesh R 2010 Materials Science and Engineering: R: Reports. 68 89-113

[3] Beche A, Rouviere J L, Barnes J P and Cooper D 2013 Ultramicroscopy. 13110

[4] Beche A, Rouviere J L, Clement L and Hartmann J M 2009 Appl.Phys.Lett. 95123114

[5] Ozdol V B, Gammer C, Jin X G, Ercius P, Ophus C, Ciston J and Minor A M 2015 Appl.Phys.Lett. 106253107

[6] Murakami Y, Sasaki T T, Ohkubo T and Hono K 2015 Acta Materialia. 101101

[7] Kim S, Jung Y, Kim J J, Lee S, Lee H and Kondo Y 2014 AIP Advances. 4107107

[8] Kim S, Lee S, Oshima Y, Kondo Y, Okunishi E, Endo N, Jung J, Byun G, Lee S and Lee K 2013 Appl.Phys.Lett. 102 161604

[9] Kim S, Kondo Y, Lee K, Byun G, Jung Kim Joong, Lee S and Lee K 2013 Appl.Phys.Lett. 103033523

[10] Kim S, Jung Y, Jung Kim Joong, Byun G, Lee S and Lee H 2014 Appl.Phys.Lett. 104161610

[11] Kim S, Lee S, Oshima Y, Kondo Y, Lee H, Lee K and Byun G 2013 ECS Solid State Letters. 3 Q1-3

[12] Kim S, Jung Y, Kim J J, Lee S and Lee H 2014 IEEE Electron Device Lett. 35 983-5

[13] Kim S, Lee S, Kondo Y, Lee K, Byun G, Lee S and Lee K 2013 J.Appl.Phys. 114053518

[14] Kondo Y and Okunishi E 2014 Microscopy. 63 391-5

[15] Su D and Zhu Y 2010 Ultramicroscopy. 110 229-33

[16] Ishizuka A, Hytch M and Ishizuka K 2017 Microscopy. 66217

[17] Shu J S and Yeh C L 1989 Optical Engineering. 28287805

[18] Gustafsson M G L 2005 Proc.Natl.Acad.Sci.U.S.A. 102 13081-6

[19] Bassett G A, Menter J W and Pashley D W 1958 Proceedings of the Royal Society A: Mathematical, Physical and Engineering Sciences. 246 345-68

[20] Hirsch P B, Howie A, Nicholson R B, Pashley D W and Whelan M J 1971 Electron Microscopy of Thin Crystals, ed P B Hirsch, A Howie, R B Nicholson, D W Pashley and M J Whelan (: Butterworths)

[21] Kondo Y and Okunishi E 2014 Microscopy. 63 391-5

[22] Hÿtch M J, Snoeck E and Kilaas R 1998 Ultramicroscopy. 74131

[23] Sawa A 2008 Materials Today. 1128

[24] Blamire M G, MacManus-Driscoll J L, Mathur N D and Barber Z H 2009 Adv Mater. 21 3827-39

[25] MacLaren I, Wang L, Craven A J, Ramasse Q M, Schaffer B, Kalantari K and Reaney I M 2014 APL Materials. 2 066106 
[26] Catalan G and Scott J F 2009 Adv Mater. 21 2463-85

[27] Béa H, Gajek M, Bibes M and Barthélémy A 2008 Journal of Physics: Condensed Matter. 20434221

[28] Wang J, Neaton J B, Zheng H, Nagarajan V, Ogale S B, Liu B, Viehland D, Vaithyanathan V, Schlom D G, Waghmare U V, Spaldin N a, Rabe K M, Wuttig M and Ramesh R 2003 Science. 2991719

[29] Asanuma S, Akoh H, Yamada H and Sawa A 2009 Physical Review B. 80235113

[30] Tsubouchi K, Ohkubo I, Kumigashira H, Oshima M, Matsumoto Y, Itaka K, Ohnishi T, Lippmaa M and Koinuma H 2007 Adv Mater. 19 1711-3

[31] Herpers A, O'Shea K J, MacLaren D A, Noyong M, Rosgen B, Simon U and Dittmann R 2014 APL Materials. 2 106106

[32] Johann F, Morelli A, Biggemann D, Arredondo M and Vrejoiu I 2011 Physical Review B. 84094105

[33] Schaffer M, Schaffer B and Ramasse Q 2012 Ultramicroscopy. 114 62-71

[34] Hartel P, Rose H and Dinges C 1996 Ultramicroscopy. 6393

[35] Takeda M, Ina H and Kobayashi S 1982 J.Opt.Soc.Am. 72156

[36] Jayhoon Chung, Guoda Lian and Rabenberg L 2010 Electron Device Letters, IEEE. 31 854-6

[37] Jones L and Nellist P D 2013 Microscopy and Microanalysis. 19 1050-60

[38] Amidror I 2009 The Theory of the Moiré Phenomenon, Volume I: Periodic Layers, ed I Amidror (: Springer London)

[39] Zeller P and Guenther S 2014 New Journal of Physics. 16083028

[40] Zhu Y, Ophus C, Ciston J and Wang H 2013 Acta Materialia. 61 5646-63

[41] Amidror I 2015 Royal Society Open Science. 2

[42] Takeda M and Suzuki J 1996 J.Opt.Soc.Am. 131495

[43] Zhu Y, Ophus C, Ciston J and Wang H 2013 Acta Materialia. 615646

[44] Park D, Herpers A, Menke T, Heidelmann M, Houben L a D, R. and Mayer J 2014 Microscopy and Microanalysis. 20 740-7

[45] Sang X and LeBeau J M 2014 Ultramicroscopy. 13828

[46] Vailionis A, Boschker H, Siemons W, Houwman E P, Blank D H A, Rijnders G and Koster G 2011 Phys.Rev.B. 83 064101

[47] Gao P, Nelson C T, Jokisaari J R, Baek S, Bark C W, Zhang Y, Wang E, Schlom D G, Eom C and Pan X 2011 Nature Communications. 2591

[48] Lee J C T, Damodaran A R, Ramesh R, Martin L W and Abbamonte P 2014 Phys.Rev.B. 89214104

[49] Chu Y H, Zhao T, Cruz M P, Zhan Q, Yang P L, Martin L W, Huijben M, Yang C H, Zavaliche F, Zheng H and Ramesh R 2007 Appl.Phys.Lett. 90252906

[50] Huang Z and Desai R C 2002 Phys.Rev.B. 65205419 
[51] Desai R C, Kim H, Chatterji A, Ngai D, Chen S and Yang N 2010 Phys.Rev.B. 81235301

[52] Janovska M, Sedlak P, Seiner H, Landa M, Marton P, Ondrejkovic P and Hlinka J 2012 Journal of Physics: Condensed Matter. 24385404

[53] Chen Z H, Damodaran a R, Xu R, Lee S and Martin L W 2014 Applied Physics Letters. 104182908

[54] Kim D H, Lee H N, Biegalski M D and Christen H M 2008 Appl.Phys.Lett. 92012911

[55] Woodward P M, Vogt T, Cox D E, Arulraj A, Rao C N R, Karen P and Cheetham A K 1998 Chemistry of Materials. $103652-65$

[56] Van Aert S, Turner S, Delville R, Schryvers D, Van Tendeloo G and Salje E K H 2012 Adv Mater. 24 523-7

[57] Velickov B, Kahlenberg V, Bertram R and Bernhagen M 2007 Z. Kristallogr. 222466

[58] Samata H, Saeki Y, Mizusaki S, Nagata Y, Ozawa T C and Sato A 2009 J.Cryst.Growth. 311623

[59] Tomashpolskii Y Y, Venevtsev Y N and Zhdanov G S 1967 Kristallogr. 12252 


\section{Figures}

Fig. 1

(a)

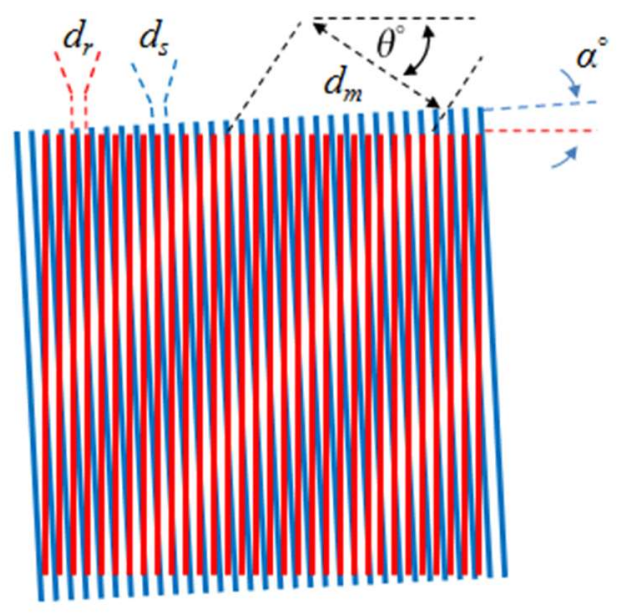

(b)

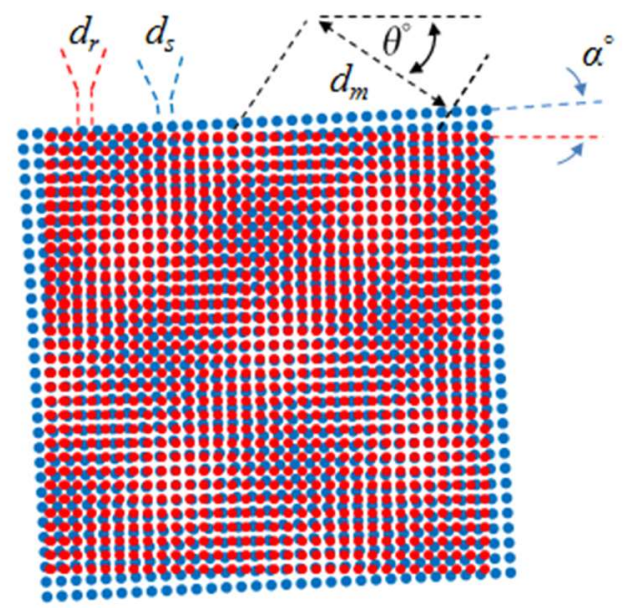

(c)

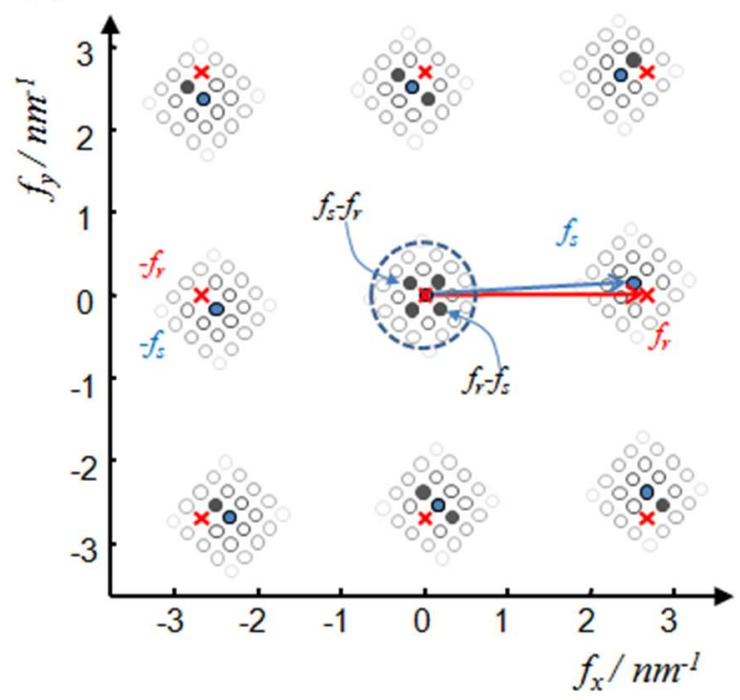

Figure 1. Overview of moiré fringe formation and analysis. (a) Two overlapping periodic patterns with fundamental periods $d_{r}$ and $d_{s}$ and set with a mutual angle $\alpha$ give rise to a low-frequency set of moiré fringes with separation $d_{m}$ and angle $\theta$. (b) Twodimensional arrays of spots produce two-dimensional moiré fringes, including fringes of the same spacing and orientation as the $1 \mathrm{~d}$ case. In both schematics, the red lines (spots) indicate the periodicity of a typical STEM scan pattern whilst the blue lines (spots) indicate the periodicity of a specimen's unit cells, scaled for the imaging of a BFO lattice at $1 \mathrm{Mx}$ magnification, aligned $3.5^{\circ}$ to the STEM scan. (c) Plotting the pattern frequencies graphically provides an intuitive analysis of moiré fringes, following the methodology presented in Ref. [20]. Here, the two dimensional array of spatial frequencies is presented but, for clarity, only one-dimensional frequencies are indexed. Red crosses indicate the frequencies of the STEM scan; blue spots indicate the frequencies of the specimen; and the moiré fringes apparent in the $1 \mathrm{~d}$ pattern arise from the vector addition $f_{m}=\left|f_{r}-f_{s}\right|$, producing two spots in the frequency plot, close to the origin. Other spots in the pattern arise from linear combinations of the reference and specimen frequencies, with decreasing amplitude as the resultant frequency increases. The dark spots derive from convolving the 4 lowest lattice frequency terms with the lowest specimen frequency terms, effectively duplicating the 4-fold pattern of blue spots around each of four other red crosses. The dotted circle indicates approximately the range of visible moire fringes in the STEM image. NB. Although the frequency plot appears like a diffraction pattern, note that a perpendicular relationship between real and reciprocal space vectors has not been adopted here. 
Fig. 2

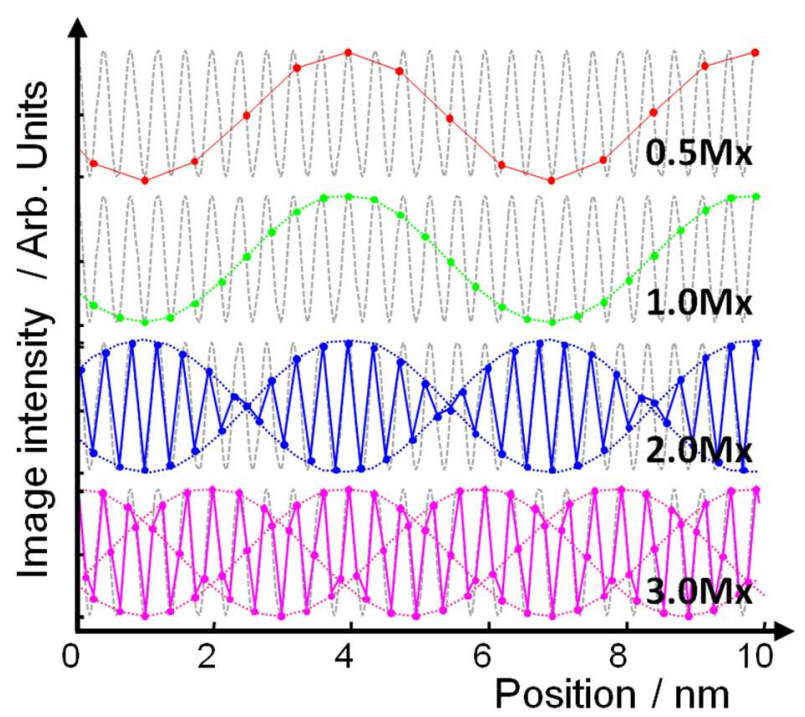

Figure 2. Calculated line profiles through STEM-moiré images of BFO at a range of magnifications. The black traces indicate the normalized image intensity along a (001)-oriented line through the A-site (Bi atoms) of the BFO perovskite cells, showing the atomic positions. The coloured overlays indicate the pixel intensity for pixels collected at each of the indicated points. At a magnification of 0.5 million times $(0.5 \mathrm{Mx})$, every other unit cell is sampled to produce what is termed here a 'second order' moiré effect. At $1 \mathrm{Mx}$ a strong moiré intensity modulation is evident. At $2 \mathrm{Mx}$ and $3 \mathrm{Mx}$, a 'fractional moiré' effects occurs and every atomic column is sampled, but with a strongly modulated pixel intensity.

Fig. 3

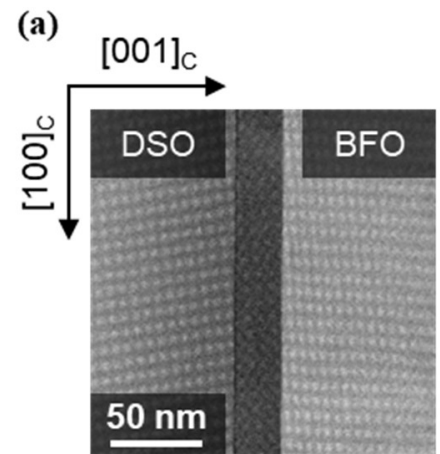

(c)

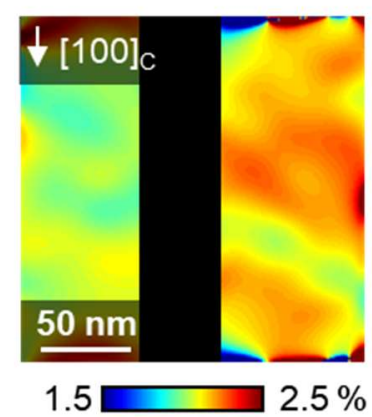

(b)

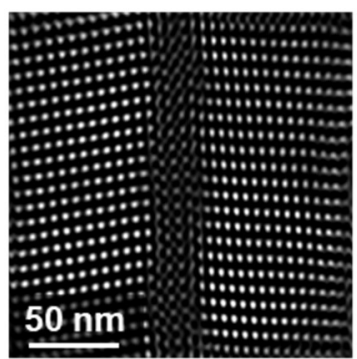

(d)

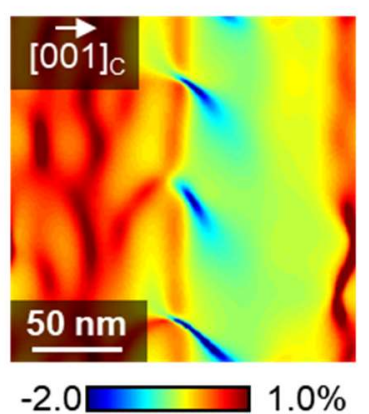

Figure 3. (a) $2 \mathrm{D}$ first order moiré image collected from the trilayer BFO sample at a nominal magnification of $1 \mathrm{Mx}$, a sampling of 512 pixels and a pixel dwell time of $77.8 \mu \mathrm{s}$. (b) Corresponding Fourier filtered fringe pattern clearly showing the 2D moiré pattern and reduced fringe visibility within the central SRO layer. (c,d) Strain maps along the fast scan axes of images such as (a). The annotations refer to the lattice spacings measured and which scan axis they correspond to. The black regions in (c) mark the SRO layer that was masked off. The strain maps are scaled with respect to the bulk BFO lattice constant. 
Fig. 4

(a)

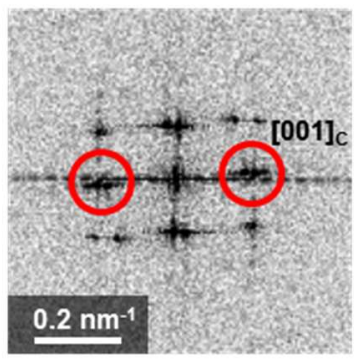

(c)

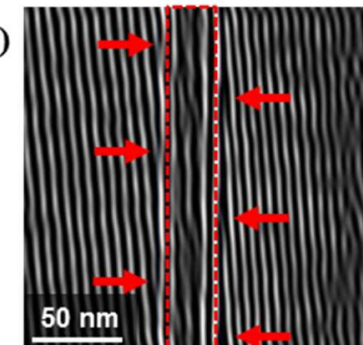

(b)

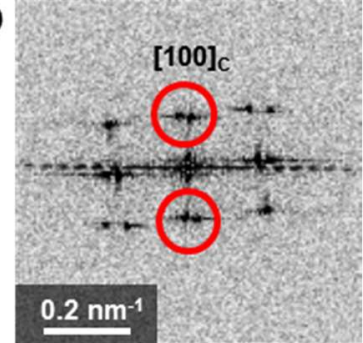

(d)

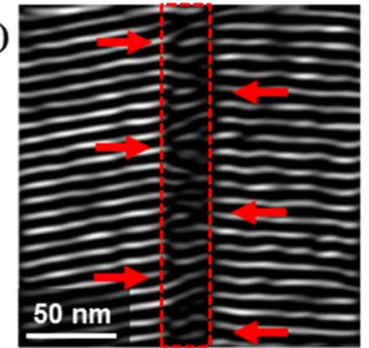

Figure 4. FFTs of raw fringes patterns from Fig. 3 with the fast scan axes aligned along (a) [001]c and (b) [100]c. The red circles depict the windows applied before calculating the corresponding inverse FFTs in (c,d), respectively. The dashed red boxes in (c,d) illustrate the location of the SRO layer and the red arrows indicate terminations of moire fringes at both the DSO/SRO and SRO/BFO interfaces. 
Fig. 5
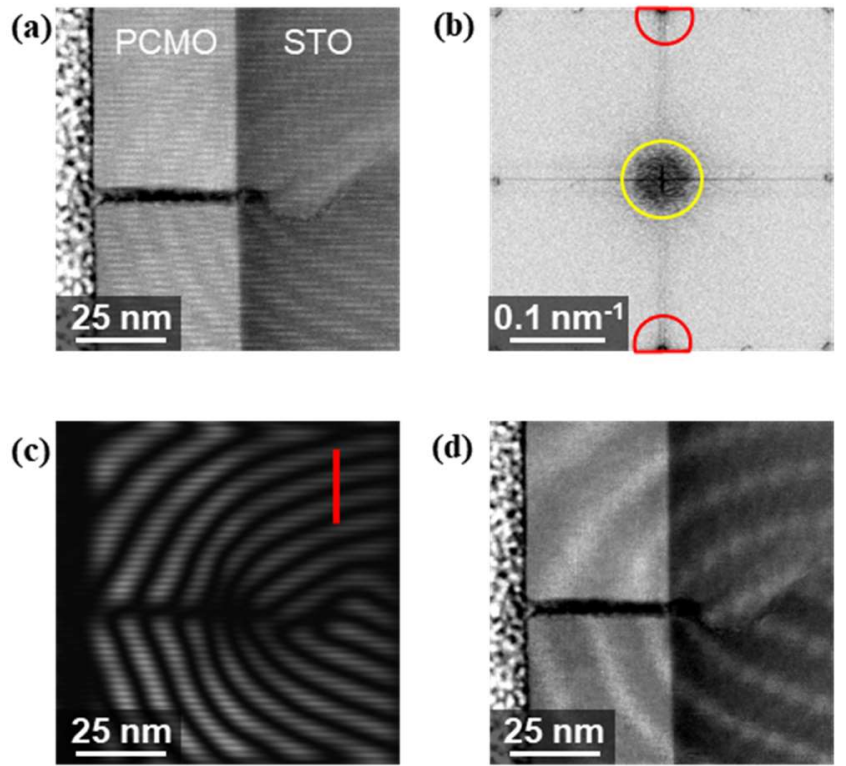

(d)

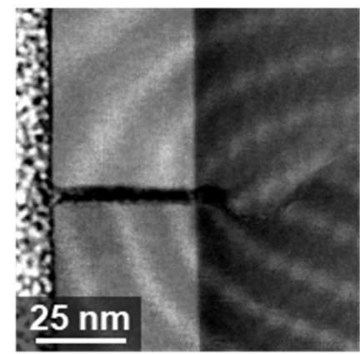

(e)

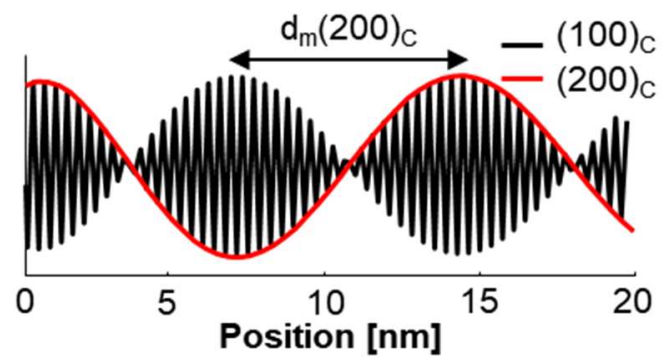

Figure 5. (a) Fractional moiré image, collected with $f_{r} \sim 2 f_{s}$, of an STO/PCMO stack. A large crack can be seen in the PCMO and STO layers and is the result of strain relaxation. Curved fringes can be seen and arise from lattice rotation. The layer along the far left edge of the image is Pt from the FIB preparation process. The slow scan axis is oriented along the vertical [100]C axis of the image. (b) FFT of (a), the red annotations show the frequencies used to produce the Fourier filtered IFFT in (c). In (c), the red annotation shows the region of the line profile in (e). (d) A (2x2) rebinned version of (a) showing low frequency fringes. (e) Line profile showing high and low frequencies, from the filtered image in $(\mathrm{c})$.

Fig. 6

(a)

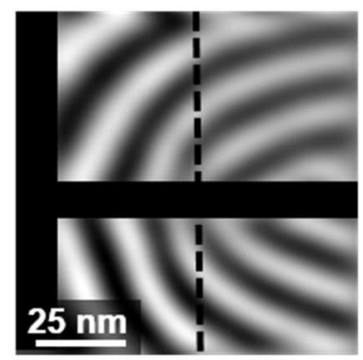

(b)

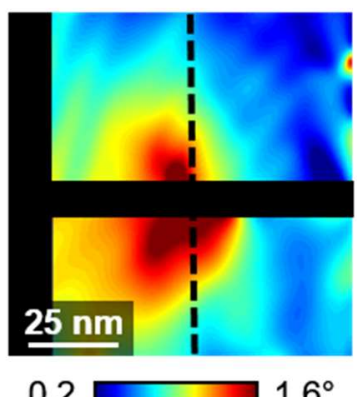

0.2

$1.6^{\circ}$

Figure 6. Moiré fringe image along the $[100]_{\mathrm{C}}$ direction, produced by rebinning and Fourier filtering the raw moiré image in figure 5. (b) Corresponding [100]c map of $|\theta|$. Masks (black regions) were applied across the Pt cap and the crack in the centre of the image to avoid errors during GPA processing. The dashed lines indicated the location of the STO/PCMO interface. $\theta$ is maximised at the base of the crack at the STO/PCMO interface in the centre of the image and gradually decreases as a function of distance. No dislocations are observed from HR-STEM [31]. 